\title{
A Simulation-Based Approach to Predict the Springback Behavior of Ultra-High Strength Spring Strips
}

\author{
Karsten Richter ${ }^{1, a^{*}}$, Franz Reuther ${ }^{1, b}$ and Roland Müller ${ }^{1, c}$ \\ ${ }^{1}$ Fraunhofer Institute for Machine Tools and Forming Technology IWU, \\ Reichenhainer Str. 88, 09126 Chemnitz, Germany \\ akarsten.richter@iwu.fraunhofer.de,bfranz.reuther@iwu.fraunhofer.de, \\ croland.mueller@iwu.fraunhofer.de
}

\begin{abstract}
Keywords: Spring strips, Bending, Stamping-bending technology, Springback prediction, FEA, Strain-dependent Young's modulus
\end{abstract}

\begin{abstract}
One effect of high influence on the dimensional accuracy during bending is springback. It inevitably occurs due to the elastic proportion in the material behavior. The impact is notably high when producing springs made of ultra-high strength spring strips of the steel grade 1.4310 (X10CrNi18-8). The high yield ratio needed to fulfil the functionalities required during application leads to dimensional inaccuracies that have to be compensated during the production process. This paper reports a simulation-based approach to predict the springback behavior of ultra-high strength spring strips with tensile strengths $T S=1500-1800 \mathrm{MPa}$. Based on the results of advanced material testing and modeling, a detailed investigation was conducted on the numerical prediction of the springback behavior of an exemplary bending process (free bending). This helps obtain deeper knowledge and understanding of the springback phenomenon and achieve suitable strategies for a more efficient industrial design for tools and processes in processing of ultra-high strength spring strips.
\end{abstract}

\section{Introduction}

Springback has been a major global research interest over the past years and decades [1]. Due to its characteristic as a highly complex phenomenon, it is a fundamental challenge to derive reliable compensation strategies based on accurate simulation-based prediction of the occurring springback. When focusing on bending, main influencing factors on the amount of springback include geometric influences (i.e. bending punches, radii and clearances, sheet thicknesses and widths), process parameters (i.e. the utilized machine, kinematics, punch and blank holder pressure) and the specific elastic-plastic material behavior of the processed materials.

Yet, there are only few published results concerning ultra-high strength spring strips. Published investigations on the springback behavior of sheet metals used for car-body components do not reach the range of tensile strengths utilized for springs. Richter et al. reported a preceding, pure experimental study on the variation of springback angles due to diverging material specifications of the steel grade 1.4310 (X10CrNi18-8) and a selective variation of process parameters [2]. The focus of the ongoing research has since been shifted to the simulation-based prediction of the springback phenomenon. The aim is to obtain deeper knowledge and understanding of the influencing parameters on the springback behavior of ultra-high strength spring strips and the specific material behavior of this steel grade. This paper specifically aims at evaluating the significance of straindependent Young's moduli $E(\varepsilon)$ for a springback prediction with FEA, which was examined and discussed for milder steels by different authors before [3-5]. For this purpose, a combined experimental and numerical study with different ultra-high strength spring strips was conducted using an exemplary bending process (free bending). 


\section{Materials}

Five different austenitic stainless steels 1.4310 (X10CrNi18-8) were included in the research. The coils were chosen with tensile strengths in the range $T S=1500-1800 \mathrm{MPa}$ with materials M1 and M3 being close to the lower limit of that range, and materials M2, M4 and M5 close to the upper limit. Sheet metal thickness was universally set at $t=0.3 \pm 0.01 \mathrm{~mm}$. The chemical composition of each of these five materials is shown in Table 1. Additionally, the allowance specified in DIN EN 10151 [6] is included.

Table 1: Chemical composition in weight percentage of the used materials

\begin{tabular}{|c|c|c|c|c|c|c|c|c|c|}
\hline & $\mathbf{C}$ & $\mathbf{S i}$ & $\mathbf{M n}$ & $\mathbf{P}$ & $\mathbf{S}$ & $\mathbf{C r}$ & $\mathbf{M o}$ & $\mathbf{N i}$ & $\mathbf{N}$ \\
\hline Allowance & $0.05-0.15$ & $\leq 2.00$ & $\leq 2.00$ & $\leq 0.045$ & $\leq 0.05$ & $16-19$ & $\leq 0.80$ & $6-9.5$ & $\leq 0.11$ \\
\hline M1 & 0.096 & 1.020 & 1.070 & 0.026 & 0.001 & 16.860 & 0.670 & 6.420 & 0.050 \\
\hline M2 & 0.101 & 0.920 & 1.330 & 0.029 & 0.002 & 16.630 & 0.390 & 6.700 & 0.075 \\
\hline M3 & 0.108 & 0.900 & 1.190 & 0.028 & 0.001 & 16.770 & 0.210 & 6.540 & N/A \\
\hline M4 & 0.100 & 1.210 & 1.230 & 0.025 & 0.001 & 16.800 & 0.670 & 6.600 & 0.065 \\
\hline M5 & 0.101 & 0.859 & 1.245 & 0.026 & 0.001 & 16.795 & 0.142 & 6.720 & 0.060 \\
\hline
\end{tabular}

\section{Experimental}

Tensile tests. In order to establish a data base for the derivation of flow curves and anisotropic behavior, uniaxial tensile tests were performed in accordance to DIN EN ISO 6892 [7]. For this purpose, stamped samples oriented at $0^{\circ}, 45^{\circ}$ and $90^{\circ}$ in relation to the rolling direction had been produced and tested under constant test conditions with at least three repetitions by default. The testing strain rate was set at $d \varphi / d t=0.000251 / \mathrm{s}$. Table 2 summarizes the basic mechanical properties (tensile strengths $T S$, yield strengths $Y S$ and ultimate elongations $U E$ ).

Table 2: Mechanical properties of the used materials

\begin{tabular}{|l|c|c|c|}
\hline & TS [MPa] & YS [MPa] & UE [\%] \\
\hline M1 & $1503-1513$ & $1328-1354$ & $8.0-12.0$ \\
\hline M2 & 1804 & 1550 & 2.0 \\
\hline M3 & $1537-1577$ & $1407-1469$ & $5.5-9.6$ \\
\hline M4 & $1729-1743$ & $1546-1556$ & $2.3-2.6$ \\
\hline M5 & $1746-1793$ & $1632-1671$ & $0.8-1.2$ \\
\hline
\end{tabular}

Cyclical tensile tests. The tensile tests were adapted in order to reach an additional strain-dependent material characterization for the numerical simulation. This especially aims at the strain- dependent reduction of the Young's modulus. Instead of a continuous and steady load, this test setup comprised a cyclical load and full unload of the stamped samples with $d \varphi / d t=0.00025 \mathrm{1} / \mathrm{s}$.
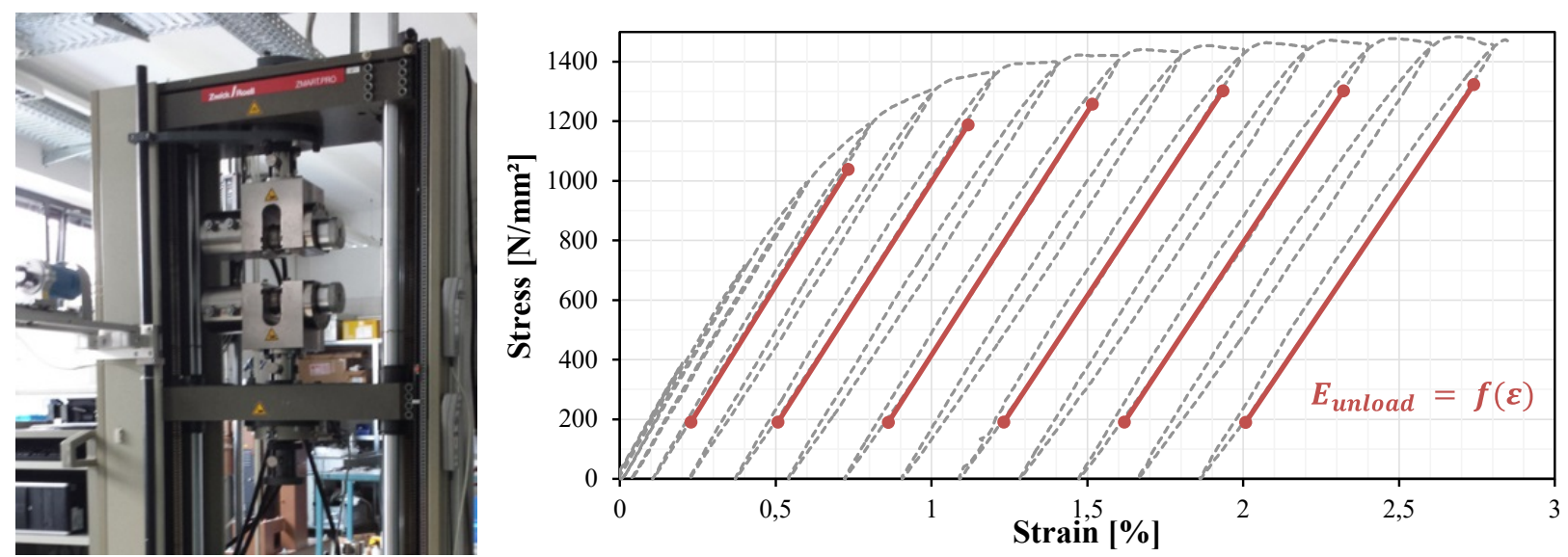

Fig. 1: Stress-strain-diagram of cyclical tensile tests 
With each cycle, the targeted strain values were increased by $\Delta \varepsilon=0.2 \%$ (Fig. 1). The small step size $\Delta \varepsilon$ was chosen with regard to the ultimate elongations $U E$ available.

The unload paths of the recorded stress-strain-hystereses of each cycle are of very high significance for the springback prediction. The extremely nonlinear area between load and unload was excluded for the analysis of the Young's moduli $E(\varepsilon)$.

Bending tests. Bending tests were executed with a small die that can be adapted to different machines (Fig. 2). Just like most dies in spring industry, it is equipped with a spring-loaded blank holder (seven pressure springs). Interchangeable bending punches with different nominal bending angles $\alpha_{n o m, i}\left(45^{\circ}, 60^{\circ}, 75^{\circ}\right)$ and radii $R_{b, i}(0.5 \mathrm{~mm}, 1.5 \mathrm{~mm}, 3.0 \mathrm{~mm})$ allow an evaluation of differing bending conditions. The bending specimens each contain a leg with a flat length of $l=10 \mathrm{~mm}$ and a width of $w=5 \mathrm{~mm}$. They were prepared by stamping with regard to their orientation to the rolling direction $\left(0^{\circ}, 90^{\circ}\right.$ and 45$)$ of the coils.

A Zwick universal testing machine was used to realize lab scale conditions suitable for the recording of load-path-curves. All bending tests were performed with a constant transversal velocity of $v=0.04 \mathrm{~mm} / \mathrm{s}$ with a termination condition set at $F=5000 \mathrm{~N}$.

Afterwards, all bent bending specimens were measured with a 3D tactile measurement system Zeiss Prismo $7 \mathrm{~S}$-ACC VAST with a probing force of $F_{p}=100 \mathrm{mN}$. Results of the measuring were angles $\alpha_{S b}$ after release. Compared to the nominal angle $\alpha_{n o m}$ of the tool active parts, the measured springback angles $\Delta \alpha$ can be calculated by a simple subtraction (for the definition of the angles cf. Fig. 2).

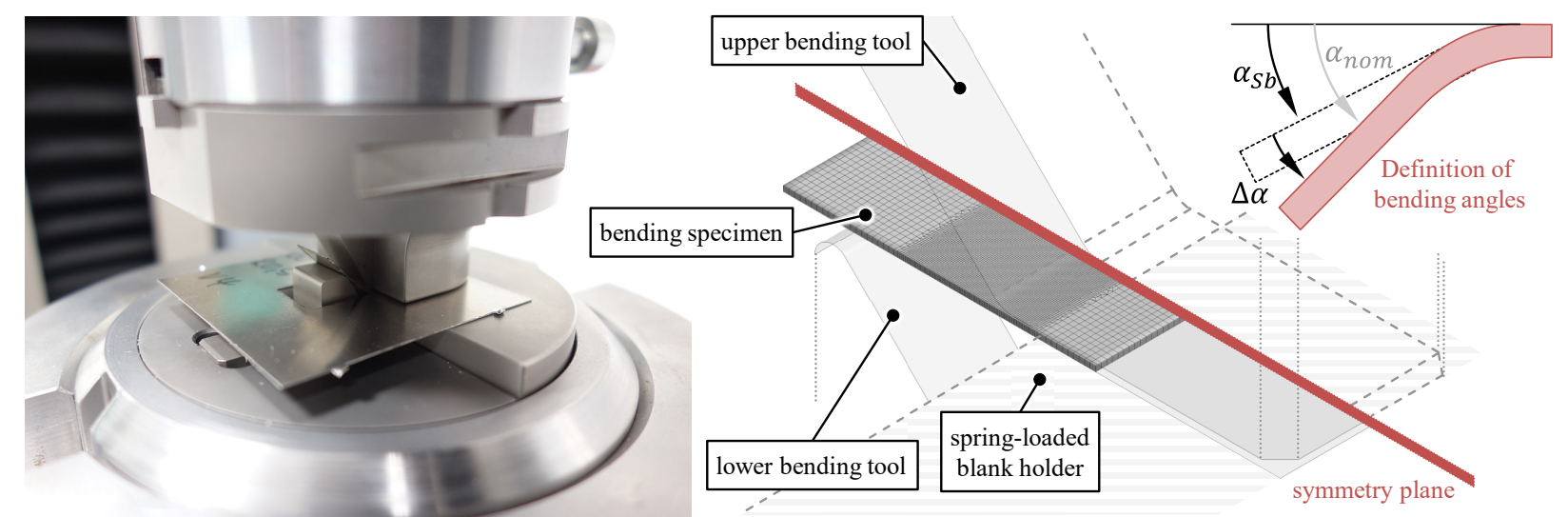

Fig. 2: Bending tests

\section{Modeling}

Modeling of the bending process. Based on the surfaces of the CAD design of the bending tool, the tool active parts and the bending process were modeled in the FE software LS Dyna. The physical properties and kinematics were implemented in accordance with the experimental set-up. This applies both to the geometries of the bending tools and to all applicable process parameters. Exploiting the symmetry, only half the geometry of the bent legs was modeled for reducing the numerical computing time.

Fig. 3 illustrates an impression of the bending process. The upper and lower bending tools and the bending specimens are shown in the initial position at the beginning of bending, in the bottom dead center at the end of bending, and after emerging springback due to release. 


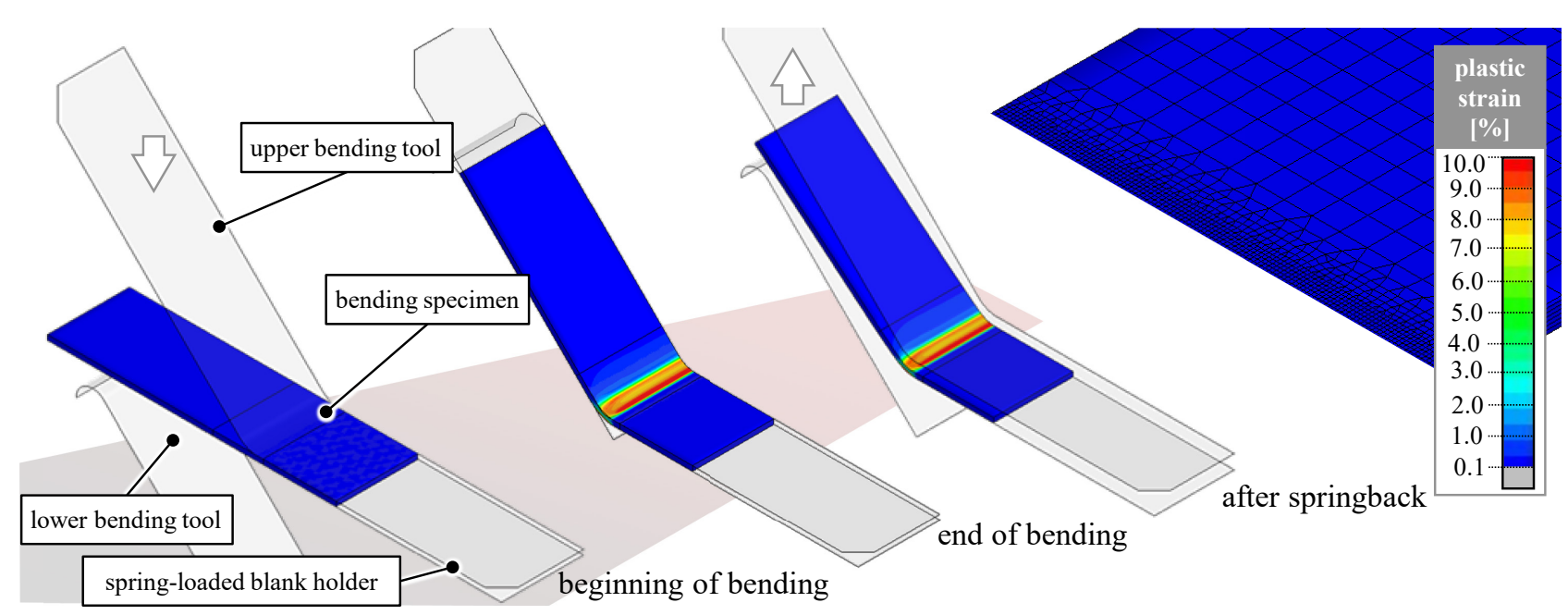

Fig. 3: FEA of the bending process

Material modeling. Using the experimental data from uniaxial tensile tests, flow curves were generated and subsequently approximated using the combined approach of Swift \& Hockett-Sherby (Fig. 4). Additionally, the Lankford coefficient ( $R$-value) describing the anisotropic material behavior was determined based on the measured elongations in width of the tensile test specimens. The yield stresses in the three tested orientations $\left(0^{\circ}, 45^{\circ}\right.$ and $90^{\circ}$ with regard to their orientation to the rolling direction) and the related $R$-values were integrated into an elastic-plastic material model. The yield locus was described according to the approach of Barlat 2000 [8]. In absence of sufficient data, the biaxial yield stress was assumed as an equivalent von Mises yield criterion.

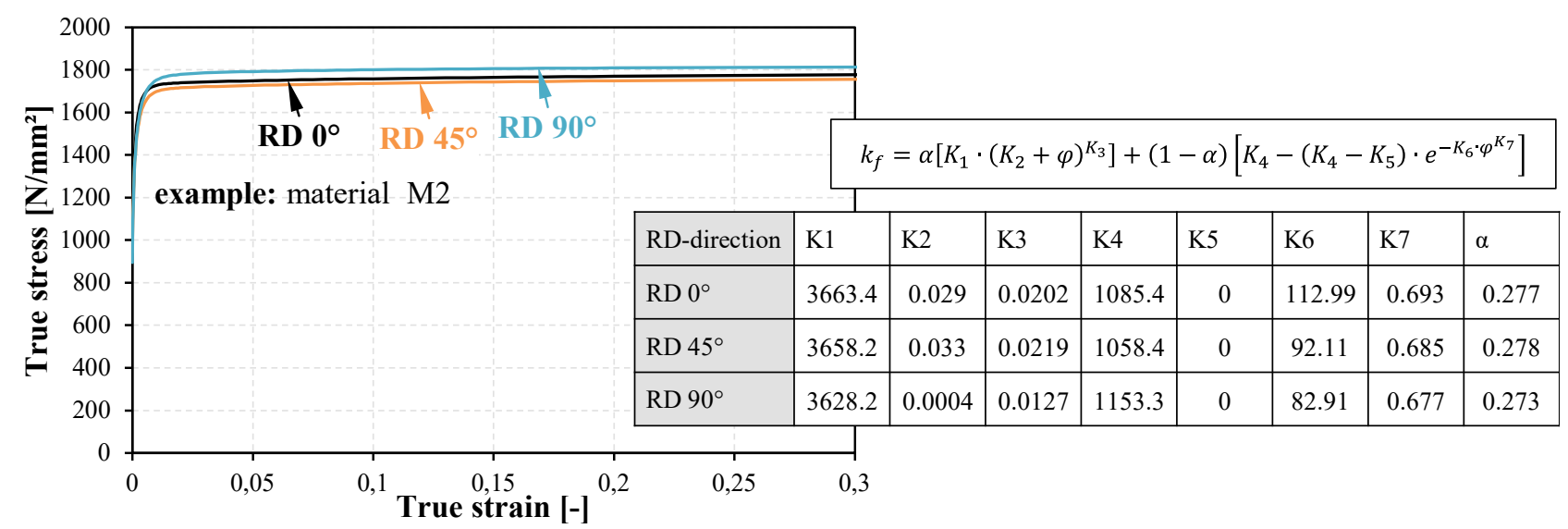

Fig. 4: Example of flow curve approximation for material M2

For loading during bending, constant values of the Young's moduli $E$ were implemented as identified in the conventional quasi-static uniaxial tensile tests. Two different modeling strategies were chosen for the release, which can be considered decisive for the evaluation of springback: In the first case, the conventional Young's moduli $E$ were retained. In the second case, straindependent Young's moduli $E(\varepsilon)$ were included as derived from the unload paths of the cyclical tensile tests.

FEA. In order to eliminate possible negative influences on the results due to mass scaling or other dynamic effects, FEA was conducted with implicit numerical time integration. The mesh of the bending specimen received a uniform discretization with an edge length of $l=0.043 \mathrm{~mm}$ in the bending zone, i.e. the sheet thickness $t=0.3 \mathrm{~mm}$ was divided into 7 elements.

Numerical studies during modeling dealt with the question whether shell elements were suitable for precise prediction of springback. This necessity primarily arose from limited options in LS Dyna for the implementation of a complex, anisotropic material model using volume elements. Since only 
a few of the experimentally established data could be included in the elastic-plastic material behavior of the spring-strips by this method, the FEA results with volume elements showed quite large systematic errors not suitable for precise springback prediction. As a result of the described observations, numerical studies were set up on shell elements with a high number of integration points (21). This measure alone allowed implementing complex, two-dimensional material models. The systematic deviations between experimental data and the predicted springback angles could be reduced significantly by switching to shell elements.

\section{Results}

Cyclical tensile tests. During the first cycle of the cyclical tensile tests, the Young's moduli $E$ are comparable to the ones measured during the uniaxial tensile tests. A tremendous reduction of $\Delta E=25-30 \mathrm{GPa}$ becomes evident during the next few cycles which constitute the mere elastic range of the material behavior. Thus, the true strain $\varphi=0$ contains several data points which vividly illustrate the described effect. The values of the strain-dependent Young's moduli $E(\varepsilon)$ flatten once the plastic range is reached.

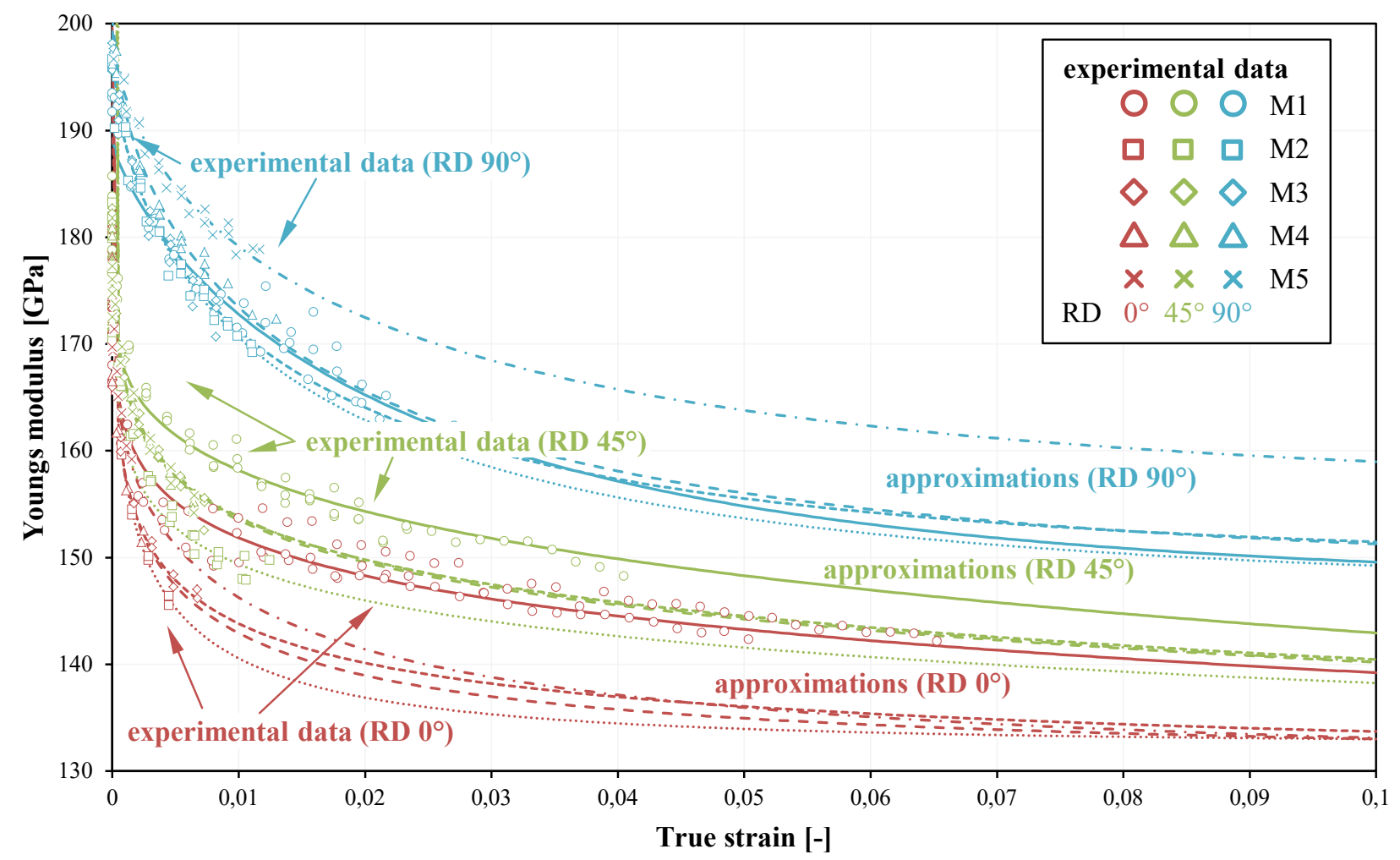

Fig. 5: Experimental data and approximation of the strain-dependent Young's moduli $E(\varepsilon)$ during release

Fig. 5 includes all the measured data points for the five tested ultra-high strength spring strips. As clearly illustrated, a limited data base is available for the extrapolation of the curves. Only material M1, which reached a true stain $\varphi=6 \%$, offered a broader data base. The materials M2-M5 only reach lower values of the ultimate strains. This has to be attributed to the fact that the strips were cold rolled in several steps. The reduction in thickness and increase in strength ultimately results in ultimate strengths which do not even exceed $U S=2 \%$ for materials M2, M4 and M5. The straindependent Young's moduli $E(\varepsilon)$ thus are experimentally only covered to the point of fracture. Principally, all materials 1.4310 show a quite similar behavior. Thus, the extrapolation was executed in assumption that materials M2-M5 show a similar progression as material M1. This comprise an imminent source of error for the following FEA that can only be overcome by using multiaxial bending tests instead of uniaxial tensile tests for material testing. This allows a collection 
of data beyond the ultimate strains which proved to be the limiting factor during the tensile tests. A possible test setup using 3-point bending was described by C. Mertin et al. [9]. Alternatively, a modification of cyclic bending tests [10-11] suitable for thin strips could be set up. Both approaches require an inverse elasto-plastic material parameter identification.

Furthermore, noticeable specific characteristics of the materials need to be discussed: Principally, there is quite a large distinction when comparing the orientation of the test samples to the rolling direction of the coils. For the orientations $0^{\circ}$ and $45^{\circ}$, the reduction of the strain-dependent Young's moduli $E(\varepsilon)$ of the materials M2-M5 corresponds quite well to each other. A bit of an outlier is material M1 with a $\Delta E(\varepsilon) \approx 5-10 \mathrm{GPa}$ compared to the other four materials. In contrast, for the orientation $90^{\circ}$ in relation to the rolling direction, material M5 shows values which are $\Delta E(\varepsilon) \approx 10 \mathrm{GPa}$ above the values of materials M1-M4. The high yield ratio YS/TS and the low ultimate elongation $U E$ contribute as main factors to the large uncertainties of the progression of the material M5.

Based on a brief literature review, two basic theories were found explaining the decrease of the Young's modulus with advancing deformation: The first theory states that the TRIP effect [12] forms the basis for the change of mechanical properties, including the Young's modulus. All the examined spring strips constitute meta-stable austenitic phases that include both $\gamma$-austenite and $\varepsilon$ and $\alpha$ '-martensite [13-14]. The specific tendency ("meta-stability") to transform unstable austenite into martensite due to strain-induction depends on the exact chemical composition which has to be taken into account for each sheet individually yet shows but small differences between the five examined materials. The diminution of the Young's moduli is further progressed due to the applied deformation during the cyclical tensile tests and the resulting higher strain hardening. The second theory emphasizes that the dislocation behavior is of higher importance to the reduction of the Young's modulus than phase transformations [3-4, 15]. Mendiguren et al. [3] concluded that microplastic strains are the main reason for this effect. Plastic deformation leads to a movement of dislocations that eventually pile up on the grain boundaries. At the same time, new dislocations appear. During stress release, unloading dislocations trending towards an equilibrium state produce extra strains that cause the non-linear unloading path. Furthermore, classic interatomic plane relocations occur during unloading.

Experimental and FEA results, discussion. The described bending process was simulated systematically with FEA. For each of the FEA runs, both the springback angles $\Delta \alpha_{F E}$ of the legs and the load-path-curves are available as results. Fig. 6 exemplarily summarizes the experimental springback angles $\Delta \alpha$ and the FEA results $\alpha_{S B}$ for two combinations of bending radius $R_{b} /$ bending angle $\alpha_{n o m}$ and for all five materials M1-M5 included in the research. In each case, the three grouped columns represent the orientation of the bending axis in relation to the rolling direction of the strips. The defined order is transversal, longitudinal and diagonal compared to rolling direction.

Well-known general tendencies can be seen both in the experimental bending tests and the FEA runs. Growing yield and tensile strengths and reduced Young's modulus lead to increased springback. The same is true for increased bending radii $R_{b}$. The bending radius $R_{b}=3.0 \mathrm{~mm}$, which represents a $R / t$ ratio of 10 , showed springback values of up to $\Delta \alpha \approx 25^{\circ}$ when anticipating a nominal angle $\alpha_{n o m}=60^{\circ}$. This is attributed to the rather low strain hardening during the bending process due to the geometrical conditions.

Generally, material M1 shows the least springback of the five experimentally examined materials. As this material has the lowest tensile and yield strengths while reaching considerably higher ultimate strengths than the other four materials, this mainly seems to be attributed to the specific mechanical properties. In spite of having tensile strengths that differ by more than $\Delta T S=220 \mathrm{MPa}$, the springback values of materials M2 and M3 are quite similar, as are the springback values $\Delta \alpha$ of the materials M4 and M5. In all cases, the orientation transversal in relation to the rolling direction shows the least springback while the values of the orientations longitudinal 
and diagonal in relation to rolling direction are quite similar. Frequently but not in all cases, the diagonal orientation shows slightly higher springback values $\Delta \alpha$ than the transversal orientation.

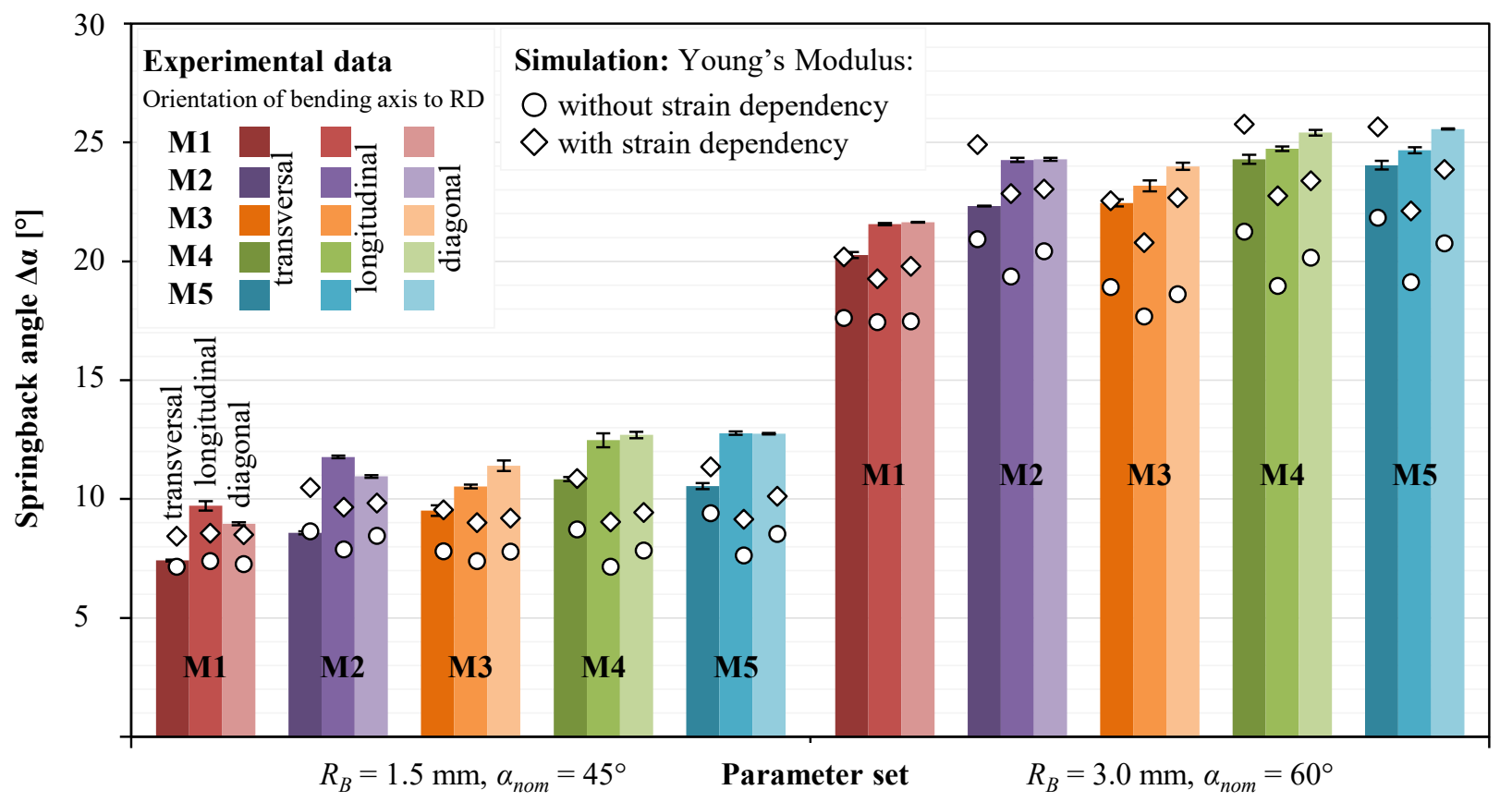

Fig. 6: Comparison of springback angles from experimental and FEA results

Table 3: Relative discrepancy of springback angles from experimental and FEA results

\begin{tabular}{|c|c|c|c|c|c|c|}
\hline $\begin{array}{l}\text { Young's Modulus in unloading } \\
\text { simulation: }\end{array}$ & M1 & M2 & M3 & M4 & M5 & average \\
\hline Without strain dependency & $16.2 \%$ & $16.5 \%$ & $23.5 \%$ & $26.1 \%$ & $22.3 \%$ & $\mathbf{2 0 . 9 \%}$ \\
\hline With strain dependency & $8.4 \%$ & $12.1 \%$ & $8.4 \%$ & $12.6 \%$ & $13.3 \%$ & $\mathbf{1 1 . 0 \%}$ \\
\hline
\end{tabular}

Comparing experimental and FEA results (Table 3), the median relative discrepancy $e$ between the observed and the predicted springback angles was $e=16.2-26.1 \%$ when considering conventional Young's moduli $E$ with an average value $e_{a v}=20.9 \%$. The relative discrepancy was calculated as the quotient of the deviation between the predicted springback angle $\triangle \alpha_{F E M}$ and the measured springback angle $\Delta \alpha$. The precision of the springback prediction in absolute values thus was $\Delta \alpha_{F E M / S B} \approx 2-4^{\circ}$. In almost all cases, the amount of springback predicted with this approach was lower than the springback seen during the experimental bending tests. Ignoring all other possible influencing factors, e.g. too idealistic modeling assumptions such as inelastic tools, this implies that the implemented Young's moduli during release had likely been oversized.

A significant improvement of the accuracy of FEA was reached when including strain-dependent Young's moduli $E(\varepsilon)$ into the material cards. The median relative discrepancy could be reduced by half to $e=8.4-13.3 \%$ with an average value $e_{a v}=11.0 \%$. Depending on the specific material and parameter set, predicted springback angles thus converge to $\Delta \alpha_{F E M / S B} \approx 0.75-2^{\circ}$ to the springback angles $\Delta \alpha$ seen during experimentation. These results clearly illustrate that strain-dependent Young's moduli $E(\varepsilon)$ deserve closer attention during any springback prediction.

Taking a closer look into the results within a specific orientation of the bending axis in a specific material, it has become clear that the observed results are somewhat insufficient. The experimental data shows that bending transversal to the rolling direction generally results in lower springback values $\Delta \alpha$ than bending in longitudinal or diagonal direction (with a maximum difference of $\Delta \alpha_{S B / F E M}=4^{\circ}$ ). A projection of those specifics with FEA has not succeeded so far as in all cases springback for bending in longitudinal direction was predicted to have the lowest values. 
The fact that so far it has not been possible to reach an even enhanced precision might well attribute to the discussed uncertainties resulting from the extrapolation of the curve progressions of the strain-dependent Young's moduli $E(\varepsilon)$ beyond the measured data points. This demonstrates the necessity to further enlarge the experimental data base aiming at an increased validation of the assumptions met.

The generation of the implemented material model might be another source of error. In the chosen approach, tensile tests were used to generate the data base for the elastic-plastic material behavior, including anisotropy. In contrast to this characterization under a uniaxial load, the examined bending constitutes a complex, biaxial strain state. Thus, the yield criterion is a mere approximation that can result in systematic deviations in the numerical results of the plastic strain during bending simulations. Likewise, the implemented Young's moduli $E$ and $E(\varepsilon)$ were generated with uniaxial tests which do not necessarily describe the specific characteristics during bending in a precise manner. Thus subject of the ongoing research is a calibration of the implemented material models by using bending tests.

The precision of the springback prediction of ultra-high strength spring-strips reached with the reported research is considerably improved compared to the standard empirical or analytical methods that have so far been established in the spring industry. Improved precision of the springback prediction results in a reduction of cost-intensive reworking steps of tools or tool active parts during toolmaking, which is a benefit that has to be valued high for spring makers.

\section{Summary and Outlook}

This combined experimental and numerical study examined the springback occurring during bending operations of ultra-high strength spring strips. Five different materials of the steel grade 1.4310 (X10CrNi18-8) with tensile strengths $T S=1500-1800 \mathrm{MPa}$ were examined concerning their specific springback behavior during an exemplary bending process (free bending). A focus had been set on the examination of strain dependencies of the Young's modulus on the precision of the numerical springback prediction. For this purpose, conventional and cyclical tensile tests were executed to generate the data base for the material model that constituted flow curves, $R$-values and either constant Young's moduli $E$ or strain-dependent Young's moduli $E(\varepsilon)$. A comparison of the experimental and the numerical results shows considerably improved precision of the numerical springback prediction when including strain-dependent Young's moduli $E(\varepsilon)$ in the material model.

The ongoing research will be continued with a calibration of the implemented material models. For this purpose, the ultra-high strength spring-strips will be characterized by bending tests that are expected to further enhance the accurate description of the mechanical material behaviors of the examined materials and the precision of the springback prediction afterwards.

\section{Acknowledgement}

The work presented in this paper has kindly been funded by the Arbeitsgemeinschaft industrieller Forschungsvereinigungen „Otto von Guericke“(grant number: IGF 19273 BR). The project has been accompanied by the German Association of Spring Manufacturers (VDFI).

\section{References}

[1] R.H. Wagoner, H. Lim, M.-G. Lee, Advanced Issues in springback, Int. J. Plast. 45 (2013) 3-20.

[2] K. Richter, F. Reuther, R. Müller, D. Landgrebe, Investigating the Influence of Bending Parameters on the Springback Behavior of Ultra-High Strength Spring Strips, Mater. Sci. Forum 918 (2018) 125-133.

[3] J. Mendiguren, F. Cortés, X. Gómez, L. Galdos, Elastic behaviour characterisation of TRIP 700 steel by means of loading-unloading tests, Mater. Sci. Eng. A 634 (2015) 147-152. 
[4] R. Pérez, J.A. Benito, J.M. Prado, Study of the Inelastic Response of TRIP Steels after Plastic Deformation, ISIJ Int. 45 (2005), 12, 1925-1933.

[5] S. Vitzhtum, M. Eder, C. Hartmann, W. Volk, Investigation on strain dependent elastic behavior for accurate springback analysis, J. Phys. Conf. Ser. 1063 (2018) 012118.

[6] DIN EN 10151, Stainless steel strip for springs - Technical delivery conditions, 2003.

[7] DIN EN ISO 6892-1, Metallic materials - Tensile testing - Part 1: Method of test at room temperature, 2017.

[8] F. Barlat, J.C. Brem, J.W. Yoon, K. Chung, R.E. Dick, S.H. Choi, F. Pourboghrat, E. Chu, D.J. Lege, Plane stress yield function for aluminium alloy sheets - Part 1: Theory, Int. J. Plast. 19 (2003) 297-319.

[9] C. Mertin, K. Huse, G. Hirt, Inverse modelling approach in 3-point bending for elasto-plastic material parameter identification of thin spring steel, J. Phys. Conf. Ser. 734/3 (2016), 032011.

[10] M. Rosenschon, S. Suttner, M. Merklein, Validation of kinematic hardening parameters from different stress states and levels of plastic strain with the use of the cyclic bending test, Key Eng. Mater. 639 (2015) 385-392.

[11] J. Franeck, Bestimmung elastisch-plastischer Materialparameter von FlugzeugAluminiumblechen unter niederzyklischen Zug-Druck-Beanspruchungen, PhD, Dresden 2014.

[12] W. Bleck, Y. Ma, X. Guo, The TRIP Effect and Its Application in Cold Formable Sheet Steels, Steel Res. Int. 88 (2017), 10, 1700218.

[13] E. Doege, S. Kulp, C. Sunderkotter, Properties and application of TRIP-steel in sheet metal forming, Steel. Res. 73 (2002) 303-308.

[14] S. Thibaud, N. Boudeau, J. Gelin, Influence of initial and induced hardening in sheet metal forming, Int. J. Damage Mech. 13 (2002) 107-122.

[15] L. Sun, R.H. Wagoner, Complex unloading behavior: Nature of the deformation and its consistent constitutive representation, Int. J. Plast. 27 (2011) 1126-1144. 